\title{
NEOPAT: Near-Earth Object PATrol program
}

\author{
Hong-Kyu Moon ${ }^{1}$, Moo-Young Chun ${ }^{1}$, Yong-Ik Byun ${ }^{2}$, Wonyong Han ${ }^{1}$, \\ Seung-Lee Kim ${ }^{1}$, Young-Beom Jeon ${ }^{1}$, Yong-Woo Kang ${ }^{2}$ \\ ${ }^{1}$ Korea Astronomy Observatory, Taejon, 305-348, Korea \\ ${ }^{2}$ Dept. of Astronomy, Yonsei University, Seoul, 120-749, Korea
}

\begin{abstract}
In 2000, Korea Astronomy Observatory launched the NearEarth Object Patrol (NEOPAT) program. NEOPAT has conducted followup observations of NEOCP (NEO Confirmation Page) objects and discovered 52 new main-belt asteroids during the observation runs. We initiated collaboration with the Yonsei Survey Telescopes for Astronomical Research (YSTAR) team for NEO search. Wide-field of view, fast readout time, and fully autonomous data pipeline will enable us to detect and track NEOs with a high efficiency. Scheduled to begin active operations in mid-2001, our survey system is going to be the first network of robotic telescopes for NEO search with automatic access to both hemispheres.
\end{abstract}

\section{Introduction}

Among the enormous numbers of bodies orbiting the sun, only a tiny fraction of them follow paths which bring them to the near-Earth space. About 90 percent of the Near-Earth Objects (NEOs) are near-Earth asteroids (NEAs) or shortperiod comets, and the other 10 percent are long-period comets. The objective of an NEO survey is to detect these objects during their periodic approaches to the Earth, to calculate their long-term orbits, and to verify the potential threat over the next few centuries. According to Chamberlin (2001), the statistics of NEAs discovered by predominant search programs in 2000 is as follows; LINEAR: 160, NEAT: 14, Spacewatch: 27, LONEOS: 38 , Catalina: 13, others: 12. The total number of NEA is a steeply increasing function of time where the cumulative total in 2000 was 1244 , while it was only 350 in 1995.

Being aware of past impacts and their destructive power, the general public as well as the astronomical community began to take anxious interest. The collision of the comet Shoemaker-Levy 9 with Jupiter, and such Hollywood films as "Armageddon" and "Deep Impact" added to the concern. Moreover, the first orbital calculations of two NEAs, 1997 XF11 and 1999 AN10 indicated that they could impact Earth in the next 50 years. However, further study has dismissed this probability. Recently, 2000 SG344 was covered on the evening news in Korea, with an animation which depicted the extinction of the dinosaurs in an exaggerated manner. The issue of an asteroid impact has widely attracted public interest, and encouraged the National Assembly and the Korean government to support astronomers to conduct NEO search. In 2000, Korea Astronomy Observatory launched the Near-Earth Object Patrol (NEOPAT) program designated 
as National Research Lab by Ministry of Science and Technology. NEOPAT is now teamed up with YSTAR (Byun et al. 2001) for collaboration in NEO research. On the other hand, NEOPAT is conducting follow-up astrometry of newly discovered NEOs with existing facilities.

\section{Follow-up Observations}

Since early 2000, NEOPAT has been reporting on the results of follow-up observations of NEOCP (http://cfa-www.havard.edu/iau/ NEO/ToConfirm.html) objects to Minor Planet Center. The observations are carried out with the Sobaeksan Optical Astronomy Observatory (SOAO) $61 \mathrm{~cm}$ telescope and the Bohyunsan Optical Astronomy Observatory (BOAO) $1.8 \mathrm{~m}$ reflector with CCDs. The NEOCP gives access to ephemerides for newly-discovered moving objects in need of confirmation. SOAO allocates 4-5 days every month for NEO followup observation. With a PM512 CCD camera mounted on the $61 \mathrm{~cm}$ reflector, we monitor the orbit of updated NEO candidates with $17 \leq \mathrm{m}_{R} \leq 19$. The observations are made more than three times over an arc of several hours during the course of at least one night, with a typical exposure time of 200-300 sec. Although we have yet applied for BOAO 1.8m telescope time, follow-up astrometry is being performed at $\mathrm{BOAO}$ before astronomical twilight, or in bad seeing conditions in the middle of the regular observation run. Employing the $1.8 \mathrm{~m}$ telescope, follow-up observations are made for 18-20 mag NEO candidates in $\mathrm{V}$ or $\mathrm{R}$ band with $30 \sim 300 \mathrm{sec}$ exposures in binning mode.

In each image, one can determine accurate position and magnitude of a suspected NEO with ASTROMETRICA using field stars with known coordinates. The coordinates of the comparison stars are taken from the HST Guide Star Catalogue or USNO 2.0. In Table 1, we list the results of follow-up observations performed at SOAO and BOAO from January to December, 2000. The total number of observations reported to MPEC is 1060 during the season.

Table 1. Observation summary of NEOCP objects

\begin{tabular}{cccccccccc}
\hline \hline & \multicolumn{3}{c}{ SOAO (345) } & & \multicolumn{3}{c}{ BOAO (344) } & \\
\cline { 2 - 3 } $\begin{array}{c}\text { Month } \\
(2000)\end{array}$ & $\begin{array}{c}\text { No. of } \\
\text { data }\end{array}$ & $\begin{array}{c}\text { No. of } \\
\text { objects }\end{array}$ & MPEC & & $\begin{array}{c}\text { No. of } \\
\text { data }\end{array}$ & $\begin{array}{c}\text { No. of } \\
\text { objects }\end{array}$ & MPEC & & Reference \\
\hline 2 & 0 & 0 & 0 & & 42 & 6 & 1 & MPC38264 \\
3 & 35 & 9 & 4 & & 73 & 13 & 4 & MPC38972 \\
4 & 25 & 5 & 3 & & 0 & 0 & 0 & MPC39716 \\
5 & 33 & 5 & 4 & & 16 & 5 & 4 & MPC40595 \\
6 & 0 & 0 & 0 & & 66 & 6 & 4 & MPC40751 \\
9 & 13 & 3 & 2 & & 23 & 8 & 0 & MPC41267 \\
10 & 0 & 0 & 0 & & 21 & 6 & 6 & MPC41456 \\
11 & 8 & 2 & 2 & & 482 & 44 & 1 & MPC41642 \\
12 & 0 & 0 & 0 & & 337 & 60 & 2 & MPC41805 \\
\hline
\end{tabular}




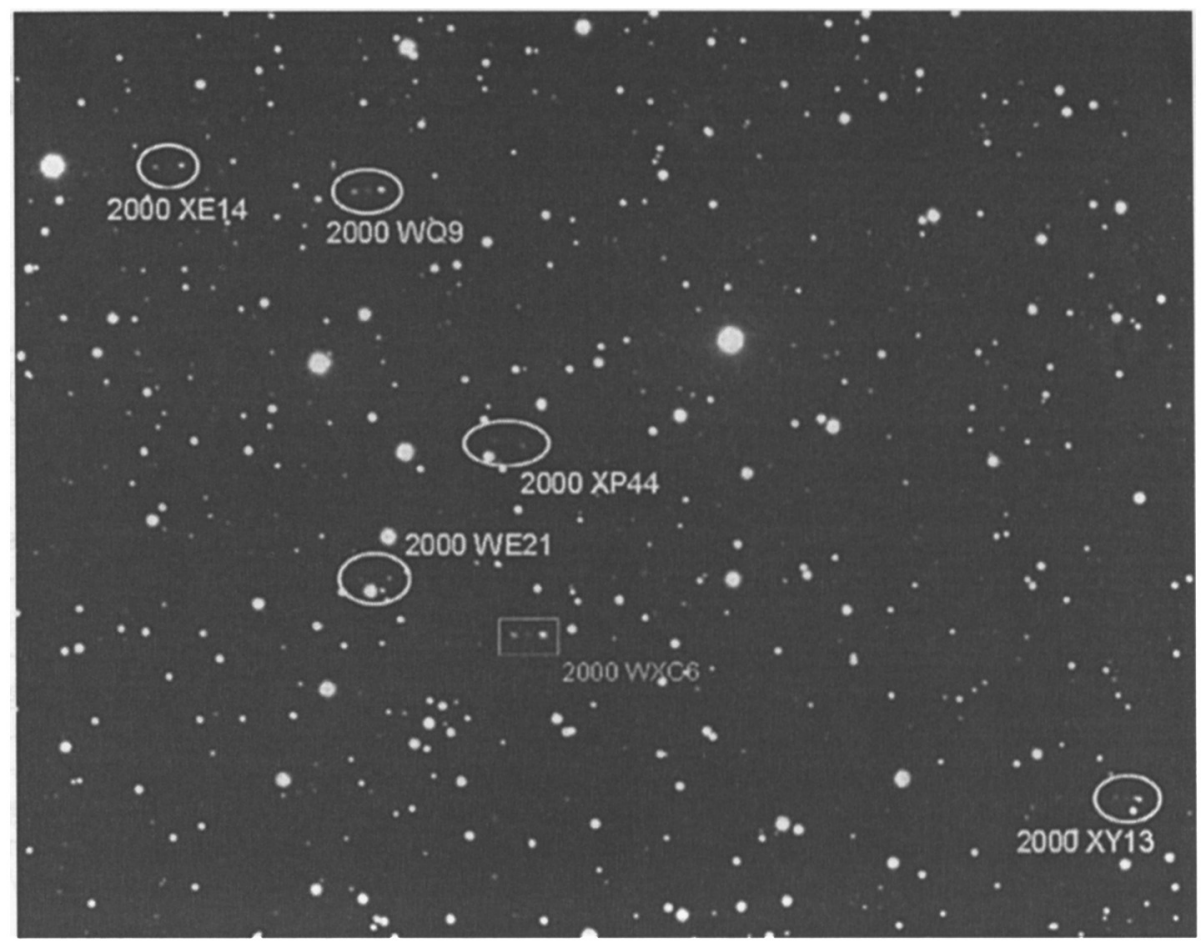

Figure 1. A CCD image obtained at BOAO on Dec. 7, 2000. Five new asteroids and one previously known asteroid are displayed in the same field. This is a color-coded image made from three consecutive $\mathrm{V}$ band CCD frames in which the asteroids were moving relative to the background stars.

\section{Discovery of New Asteroids}

Since May 2000, we have discovered 52 new asteroids during the follow-up observation of NEOCP objects, and also from color-coded images of the Crab nebula (M1) taken for public release. The first asteroid, $2000 \mathrm{KJ} 4$ was found on May 28,2000 , and turned out to be a new main-belt asteroid with an absolute magnitude of $(\mathrm{H}) \sim 15.6$, after confirmation observations on consecutive nights. The name, date of designations, and observers of these serendipitous discoveries are summarized in Table 2.

Figure 1 shows a discovery image of Dec 7, 2000 taken from the BOAO $1.8-\mathrm{m}$ reflector equipped with a SITe $2 \mathrm{~K}$ CCD camera. It reveals five newly discovered asteroids, $2000 \mathrm{XE14}, 2000 \mathrm{WQ9}, 2000 \mathrm{XP} 44,2000 \mathrm{WE} 21$, and 2000 XY13 marked with an ellipse, together with a known asteroid at the location shown by a box in the same field. This is a color-coded photograph made from three consecutive V band CCD frames where the asteroids were moving relative to the background stars, so they look slightly trailed with different colors. We conducted confirmation observations of the neighboring fields, and within 
Table 2. New main-belt asteriods discovered at BOAO

\begin{tabular}{|c|c|c|c|c|}
\hline \multicolumn{5}{|c|}{ Designation } \\
\hline $2000 \mathrm{KJ} 4$ & 2000 WQ9 & 2000 WE21 & $2000 \mathrm{WD} 21$ & 2000 WR21 \\
\hline 2000 WV 28 & 2000 WV 50 & 2000 WU50 & $2000 \mathrm{XY} 13$ & $2000 \times Z 13$ \\
\hline $2000 \times A 14$ & $2000 \times B 14$ & $2000 \times C 14$ & $2000 \mathrm{XD} 14$ & $2000 \mathrm{XE} 14$ \\
\hline $2000 \mathrm{XJ} 15$ & $2000 \times K 15$ & $2000 \mathrm{XL} 15$ & $2000 \mathrm{XM} 15$ & $2000 \times \mathrm{XA} 44$ \\
\hline $2000 \times B 44$ & $2000 \times C 44$ & $2000 \mathrm{XD} 44$ & 2000 WZ26 & $2000 \mathrm{XJ} 2$ \\
\hline 2000 XK2 & $2000 \times \mathrm{XO} 44$ & $2000 \times \mathrm{XP} 44$ & $2000 \mathrm{XQ} 44$ & $2000 \times R 44$ \\
\hline 2000 XL53 & $2000 \mathrm{XM} 53$ & $2000 \times N 53$ & 2000 XO53 & $2000 \mathrm{XP} 53$ \\
\hline 2000 XQ53 & $2000 \times T 53$ & 2000 YE04 & $2000 \mathrm{YBi} 6$ & $2000 \mathrm{YC} 16$ \\
\hline 2000 YD16 & 2000 YS30 & 2000 YDA1 & 2001 AN19 & $2001 \mathrm{AO} 19$ \\
\hline 2001 AP19 & 2001 AQ19 & 2001 AR19 & 2001 AS19 & $2001 \mathrm{BB} 03$ \\
\hline $2001 \mathrm{BC} 03$ & $2001 \mathrm{BZ} 10$ & & & \\
\hline
\end{tabular}

a month, found forty new moving objects. The newly discovered objects are also known to be main-belt asteroids with semi-major axes, $2.2 \leq \mathrm{a} \leq 3.4 \mathrm{AU}$, and ellipticities of the orbits ranging from 0.0 to 0.25 . The reasons we discovered dozens of new objects in a relatively short period of time are that: (1) December is the most favorable season for astronomical observation in Korea, and period when the ecliptic rises high above the horizon, (2) We can reach as faint as 20th magnitude, provided that we make use of the $1.8-\mathrm{m}$ telescope with a suitable exposure time. For the time being, there is no dedicated $2 \mathrm{~m}$ class telescope in regular service for asteroid search.

\section{NEOPAT-YSTAR Collaboration}

A robotic telescope is suitable for repetitive and routine work such as discovery and tracking of NEOs with maximum efficiency. Undoubtedly, a survey project can be successfully accomplished with a global network of telescopes of this kind. NEOPAT team is developing such system in collaboration with YSTAR.

We employ wide field optics and a fast, fully automated computing pipeline. The $0.5 \mathrm{~m} \mathrm{f} / 2.0$ primary optics identical to the one prepared for the TAOS project (King 2001), provides 3.5 square degrees FOV onto an AP10 $14 \mu \mathrm{m}$ CCD chip. The telescopes are being subjected to remote observation and safety tests. Our first telescope has just begun remote autonomous observations at Yonsei University Observatory, and after its launch in mid 2001, we plan to install the second telescope in Sutherland, at the South African Astronomical Observatory (SAAO). Before the SAAO installation, the NEOPAT-YSTAR team will complete the fine-tuning and development of the 3TB data storage system, the data pipeline, the observation planner/scheduler, and the off-site image archiving system. We intend to archive the data at the Korea Astronomy Observatory and to build a common database for ease of future analysis.

In a couple of years, we will expand the number of survey telescopes and place them home and abroad to increase the detection and tracking efficiency by multiplying the coverage. As such a network of survey telescopes is expected 
to provide a huge search volume, a large number of new NEOs are expected to be found far from the ecliptic plane. Moreover, orbits of long-period comets are so elongated and inclined to the ecliptic plane that the warning time for objects of this kind would be as short as a year compared to decades or centuries for asteroids. In spite of their small apertures, our survey telescopes offer more frequent coverage of the entire sky for effective search of long-period comets.

\section{Conclusions}

The NEOPAT program has carried out confirmation observation of NEOCP objects and discovered 52 new asteroids, among them forty were found within a month. In 2000, the joint program between NEOPAT and YSTAR was launched. We have just started routine autonomous observation with our first telescope and the second one is scheduled to be installed at SAAO in summer 2001. In the coming years, a network of survey telescopes will be constructed around the globe for more efficient monitoring of NEOs.

\section{References}

Byun, Y.-I. et al. 2001, in these proceedings

Chamberlin, A. 2001, http://neo.jpl.nasa.gov/missions/stats.html King, S. K. 2001, in these proceedings 